\title{
ALÇA INTESTINAL INVERTIDA COMO SUBSTITUTO DO URETER: ESTUDO EXPERIMENTAL EM CÃES ${ }^{1}$
}

\author{
URETER REPLACEMENT BY INVERTED ILEAL SEROMUSCULAR \\ TUBE OR PATCH
}

\author{
Antonio F. D. Maynard ${ }^{2}$ \\ Antonio Carlos Pereira Martins ${ }^{3}$ \\ Tiago José Borelli-Bovo, ${ }^{4}$ \\ Haylton Jorge Suaid ${ }^{3}$ \\ Adauto José Cologna ${ }^{3}$ \\ Silvio Tucci Junior ${ }^{3}$
}

\section{RESUMO}

Objetivo - Pesquisar a viabilidade da alça ileal invertida como substituto ureteral. Métodos - Quarenta cães foram divididos em 5 grandes grupos de 8 animais, cada um subdividido em 2 subgrupos de acordo com a data do sacrifício (I - sacrifício em 12-15 dias de pósoperatório, e II - 60 dias de pós-operatório). Nos grupos A e B fez-se a substituição ureteral parcial com selo de íleo invertido, após abertura lateral do ureter direito com 2 (Grupo A) ou $4 \mathrm{~cm}$ (Grupo B) de extensão. Nos demais grupos fez-se a substituição do segmento completo do ureter usando-se um tubo de íleo invertido de comprimento equivalente ao segmento do ureter ressecado com $2 \mathrm{~cm}$ (Grupo C) e $6 \mathrm{~cm}$ (Grupos D e E) de extensão. A mucosa ileal foi removida por raspagem ou diérese. A permeabilidade ureteral foi testada por urografia excretora e por exploração anatômica com cateter após o sacrifício. As peças foram estudas histologicamente após preparação e coloração com hematoxilina-eosina. Resultados - O número de animais com urografia normal após o seguimento foi: A-2, B2, C-3, D-2 e E- 2. A histologia revelou que a serosa ileal se reveste com urotélio, não como decorrência de metaplasia, mas do crescimento a partir da borda da mucosa ureteral. Conclusão - Conclui-se que o íleo invertido não é um bom substituto ureteral. Disponível em URL: http://www.scielo.br/acb
Descritores - Substituto ureteral, tubo ileal seromuscular, lesão de ureter.

\begin{abstract}
Objective - To investigate the outcome of ureter replacement with inverted seromuscular tube in dogs. Methods - 40 adult mongrel dogs were divided in 5 groups of 8 animals. Each group was divided in 2 subgroups: 1 - sacrifice on 12-15 postoperative days, and 2 - sacrifice on the $60^{\text {th }}$ postoperative day). In group A ureter longitudinal incision $2 \mathrm{~cm}$ long was sewed with an inverted ileal seromuscular patch $2 \mathrm{~cm}$ long. In group B ureter incision and patch size were $4 \mathrm{~cm}$ long. In the remaining groups a ureter segment was replaced by an inverted ileal seromuscular tube $2 \mathrm{~cm}$ long (group C) or $6 \mathrm{~cm}$ long (groups D and E). In all groups, except $\mathrm{E}$, ileal mucosa was removed by a grasping maneuver with a scalpel. In group $\mathrm{E}$ the ileal mucosa was trimmed away by blunt dissection with a scissors. The ureter permeability was tested by an intravenous urogram, and after sacrifice by anatomic exploration with a catheter. All ureter segment removed through the necropsy were analyzed by histology after hematoxilin-eosin coloration. Results - The number of animals with normal urogram after the followup was: $\mathrm{A}-2, \mathrm{~B}-2, \mathrm{C}-3, \mathrm{D}-2$ and $\mathrm{E}-2$. Histology revealed that ileal serosa was covered by ureteral mucosa grown
\end{abstract}

\footnotetext{
1 Trabalho realizado no Departamento de Cirurgia e Anatomia - Faculdade de Medicina de Ribeirão Preto-USP.

2 Professor Adjunto, Faculdade de Medicina da Universidade Federal de Sergipe.

Docentes da Divisão de Urologia da FMRP-USP.

Médico Residente do HCFMRP-USP.
} 
from the ureter buds. Conclusion - The inverted ileal seromuscular tube or patch does not work well for ureter replacement.

Key words - Ureter replacement, ileal seromuscular tube, ureter injury

\section{INTRODUÇÃO}

Na prática urológica deparamo-nos não raramente com perdas ureterais sérias. Para evitar a nefrectomia tem-se tentado nos últimos 90 anos o reparo ou a substituição do uterer por próteses, tecidos biológicos tubulares ou não e autotransplante $e^{1,2,3,4}$.

Dentre as próteses, várias substâncias sintéticas foram testadas como o polietileno ${ }^{5}$, o silicone ${ }^{2}$, o teflon ${ }^{6}$, e o gore-tex ${ }^{7}$, com resultados variáveis mas geralmente insatisfatórios devido a cristalização de sais, deiscência das anastomoses, ausência de peristaltismo ou obstrução.

Vários tecidos biológicos não vascularizados também foram testados com resultados insatisfatórios: veia safena $^{3}$, segmentos arteriais ${ }^{8}$, segmentos ureterais autólogos e homólogos ${ }^{1}$ e esponja de colágeno ${ }^{9}$. Resultados mais animadores foram conseguidos com mucosa vesical tubulizada para substituição de segmentos de até $1,5 \mathrm{~cm}$ de ureter ${ }^{4}$, com selos de peritôneo ${ }^{10} \mathrm{e}$ veia umbilical heteróloga $a^{11}$.

Dentre os tubos biológicos vascularizados a trompa de Falópio não ofereceu bons resultados ${ }^{12}$ enquanto os obtidos com o apêndice cecal são controvertidos ${ }^{13}$. Um método efetivo e capaz de substituir o ureter em sua porção distal é pela tubulização de um retalho vesical conhecido pelo nome de técnica de Boari, mas proposta originalmente por Van $\mathrm{Hook}^{14}$. O uso de alça ileal para substituição ureteral foi proposto inicialmente por Fenger (1894) mas primeiramente realizada por Boari (1894) em cães ${ }^{15}$. Mas, foi somente na década de 1950 que esta técnica se consolidou como método válido para substituição parcial ou total do ureter ${ }^{16,17,18}$. Entretanto, várias são as desvantagens mencionadas na literatura a respeito da utilização deste substituto ureteral, entre eles a formação de cálculos pela produção de muco, infecçao urinária de repetição, refluxo vésicoíleo-renal, estenoses e absorção de eletrólitos e catabólitos protéicos urinários ${ }^{18,19}$. Em razão dessas complicações várias modificações foram propostas, como a ressecção parcial da parede ileal para redução do calibre ileal ${ }^{20} \mathrm{e}$ a construção de válvula anti-refluxo por intussuscepção ileal ${ }^{21}$, com resultados relatados como favoráveis. Outras tentativas experimentais foram feitas, como o uso do íleo invertido ${ }^{22}$ e do tubo seromuscular ileal cruento (com remoção da mucosa) mas com resultados insatisfatórios ou controvertidos ${ }^{23}$.

Conclui-se, portanto, que ainda não se encontrou um substituto ureteral satisfatório, pois os métodos disponíveis ou apresentam complicações importantes ou não foram totalmente estudados, como é o caso do íleo invertido.O presente estudo foi realizado no intuito de verificar a viabilidade ou não desta técnica, quer como selo ou tubo de variados comprimentos.

\section{MÉTODOS}

\section{Grupos experimentais}

Quarenta cães adultos mestiços de ambos os sexos, com peso variável entre 14 e $16 \mathrm{~kg}$, foram divididos ao acaso em 5 grandes grupos de 8 animais. Cada um desses grupos foi subdividido em 2 subgrupos de acordo com a data de sacrifício (subgrupo I com sacrifício em 12-15 dias de pós-operatório, e II com 60 dias de pós-operatório). Nos grupos A e B fez-se a substituição ureteral parcial com selo de íleo invertido, após abertura lateral do ureter direito com 2 (Grupo A) ou $4 \mathrm{~cm}$ (Grupo B) de extensão. Nos demais grupos fez-se a substituição do segmento completo do ureter usando-se um tubo de íleo invertido de comprimento equivalente ao segmento do ureter ressecado com $2 \mathrm{~cm}$ (Grupo C) e $6 \mathrm{~cm}$ (Grupos D e E) de extensão. Em todos os animais excetuando os do Grupo E, antes da confecção do selo, ou tubo, a mucosa intestinal foi removida por raspagem com lâmina de bisturi. No Grupo $\mathrm{E}$ a mucosa foi removida por diérese com tesoura.

\section{Preparo pré-operatório/ anestesia.}

Todos os animais foram submetidos a jejum préoperatório de 12 horas antes da cirurgia. Aqueles sorteados para o sacrifício aos 60 dias de pós-operatório (subgrupos II) submeteram-se a urografia excretora com paraaminobenzoato sódico $50 \%, 1 \mathrm{ml} / \mathrm{kg}$ por via endovenosa, imediatamente antes do ato operatório. Para o ato cirúrgico os cães foram anestesiados por via endovenosa com nembutal sódico na dose de $30 \mathrm{mg} /$ $\mathrm{kg}$, entubados com sonda endotraqueal e submetidos a ventilação controlada.

\section{Técnica cirúrgica}

Todos os animais foram submetidos a laparotomia mediana xifo-púbica. O 1/3 médio do ureter direito foi dissecado e isolado. Em seguida, isolou-se o segmento de íleo terminal preservando-se a vascularização. $\mathrm{O}$ trânsito intestinal foi reconstruido por anastomose íleoileal término-terminal com pontos separados de algodão 
3-0. O segmento ileal isolado foi então aberto longitudinalmente na borda anti-mesentérica. A mucosa ileal foi removida por raspagem com lâmina de bisturi ou por ressecção com tesoura usando-se como referência o plano submucoso. Nos grupos A e B o ureter foi aberto longitudinalmente na extensão apropriada, e o defeito ureteral foi reconstruído com selo confeccionado com o íleo isolado previamente, usando-se pontos separados de catgut 4-0 cromado. Nos demais grupos, usou-se o íleo para confecção de um tubo modelado sobre um catéter de polietileno $\mathrm{n}^{\circ} 116$, suturado com pontos contínuos de catgut 4-0. O comprimento deste tubo foi ajustado conforme o tamanho do segmento do ureter ressecado, e em seguida o tubo foi usado para substituir o segmento ureteral removido, tomando-se o cuidado de respeitar o sincronismo peristáltico das estruturas, empregando-se pontos separados de catgut 4-0 cromado para a sutura dos cabos proximal e distal. Em todos o grupos, o íleo foi colocado em posição invertida, ou seja, a serosa ileal ficou voltada para a luz ureteral. Nos grupos C, D e E o cater de polietileno foi deixado moldando o tubo ileal. O passo final consistiu no fechamento da parede abdominal por planos. Nos subgrupos I deixou-se o catéter de polietileno moldando o ureter até o momento do sacrifício dos animais. Nos subgrupos II não se deixou catéter moldando o ureter.

\section{Pós-operatório}

Todos os animais receberam nos primeiros 5 dias trimexazol intramuscular na dose diária de $30 \mathrm{mg}$ de sulfa e $6 \mathrm{mg}$ de trimetropima por $\mathrm{kg}$ de peso. Após a cirurgia os animais receberam dieta líquida por $24 \mathrm{~h}$ e daí em diante ração comercial ad libitum.

\section{Sacrifício}

$\mathrm{Na}$ data do sacrifício os animais foram anestesiados com nembutal sódico na dose de $30 \mathrm{mg} / \mathrm{kg}$, endovenoso, e em seguida receberam éter sulfúrico por via endovenosa até a parada cardiorespiratória. Realizouse então a necrópsia para estudo da cavidade abdominal, da permeabilidade do ureter direito e obtenção de material para estudo histológico após fixação em formol a $10 \%$, inclusão em parafina e coloração em hematoxilina e eosina de secções de 5 micra de espessura.

Imediatamente antes do sacrifício, os cães dos subgrupos II foram submetidos à urografia controle.

\section{Análise estatística}

A análise de variáveis categóricas como a patência da anastomose ureteral ou existencia ou não de hidroureter foi feita pelo teste exato de Fisher, considerando-se nível de significância $p=<0,05$.

\section{RESULTADOS}

\section{Evolução clínica}

A observação diária foi semelhante quanto a recuperação anestésica, início da movimentação e aceitação de dieta nos diversos grupos.

\section{Achados macroscópicos e urográficos}

\section{Grupo $A$}

À necrópsia encontrou-se obstrução ureteral completa em 2 dos 8 animais estudados. A análise macroscópica e a urografia mostraram dilatação leve ou moderada do ureter direito em 4 animais e trato urinário normal em 2 cães, sendo 1 após 15 dias de evolução (AI) e 1 após 60 dias (AII).

\section{Grupo B}

No subgrupo BI notou-se obstrução ureteral com hidronefrose grave e abscesso retroperitoneal em 1 cão. Hidronefrose discreta foi observada em 2 animais, um dos quais apresentava deiscência parcial da anastomose uretero-ileal e coleção de urina encistada.

No subgrupo BII observou-se obstrução ureteral completa e hidronefrose em 1 animal. Nos demais, o ureter estava pérvio mas 2 dos 3 cães exibiram hidronefrose discreta sendo que uma pequena coleção de muco para-ileal. No cão restante a urografia excretora foi normal.

\section{Grupo C}

Todos os animais do subgrupo CI apresentavam ureter patente, mas em 2 deles a urografia revelou hidronefrose moderada. Um cão apresentava urografia normal. Em todos constatou-se também coleção de muco para-ileal.

No subgrupo CII ocorreu obstrução ureteral com hidronefrose grave em 2 cães e nos outros dois a urografia foi normal. Em 3 animais deste subgrupo havia coleção de muco justa ileal.

Os cateteres de polietino migraram e foram encontrados na cavidade vesical de todos animais.

\section{Grupo D}

No subgrupo DI, houve obstrução ureteral e hidronefrose em apenas um animal; nos demais, 2 apresentaram hidronefrose discreta e 1 exibiu rim direito 
normal. No subgrupo DII 1 animal apresentou obstrução ureteral com hidronefrose acentuada, 2 revelaram hidronefrose moderada e 1 rim normal.

Neste grupo o cateter de moldagem também migrou para a bexiga em $7 / 8$ animais.

\section{Grupo E}

Nenhum dos animais do subgrupo EI apresentou hidronefrose embora em 3 deles se tenha notado discreto hidroureter. No subgrupo EII, um cão apresentou exclusão renal, 1 hidronefrose grave, 1 hidronefrose discreta e 1 rim normal.

Neste grupo o cateter de polietileno migrou para a bexiga em 5 cães, em 2 a migração ocorreu para o ureter à montante e em 1 manteve-se bem posicionado moldando o tubo ileal.

\section{Exame histopatológico}

Os achados histológicos nos grupos A e B revelam infiltrado inflamatório tipo pielonefrite ou focos de infiltrado inflamatório crônico intersticial na maioria dos cães (10 em 16). Quanto à parede do selo ileal, notouse epitelização da face voltada para a luz do trato urinário em todos os cães, sendo completa em 12 e incompleta nos demais. Notou-se que a epitelização era completa nas extremidades do selo, associada a ausência ou lentidão do processo de epitelização na área central. $\mathrm{Na}$ face externa do selo, encontrou-se extensa regeneração de mucosa, com áreas císticas de muco e intenso processo inflamatório crônico em 12 animais.

Nos grupos C e D, 14 animais evidenciaram pielonefrite, não destoando dos grupos anteriores. Ocorreu epitelização total da face luminar do tubo ileal, com mais de 3 camadas de células transicionais, em 5 animais, e nos demais a epitelização foi parcial. Na face externa do tubo ileal ocorreram focos de regenaração da mucosa intestinal em 14 cães e cistos de muco.

O grupo E evidenciou epitelização parcial da luz ileal em todos os animais, sendo que externamente não ocorreu regeneração da mucosa ileal ou formação de cistos de muco.

\section{Análise Estatística}

A análise estatística comparando os diversos grupos quanto a existência de hidroureter $(\mathrm{p}>0,05)$ e a permealidade ureteral $(\mathrm{p}>0,05)$ não mostrou diferença significante.

\section{DISCUSSÃ̃o}

A reconstrução ureteral com selo invertido de alça vascularizada de $2 \mathrm{~cm}$ de comprimento não mostrou bons resultados, pois apenas 2 de 8 animais operados apresentaram trato urinário normal no pós-operatório, sendo 1 no $15^{\circ}$ dia e outro no $60^{\circ}$ dia. Como a obstrução mecânica foi demonstrada em apenas 2 cães deste grupo, depreende-se que em 6 deles havia subestenose ureteral na região do selo ou menos provavelmente obstrução parcial funcional. No grupo B, com selo invertido de $4 \mathrm{~cm}$ de extensão os resultados foram semelhantes, constatando-se trato urinário normal em apenas $3 / 8$ dos cães. Tais dados são de difícil confronto com a literatura, muito embora Kendall \& Karafin $(1969)^{24}$ tenham relatado patência em $100 \%$ dos ureteres (apesar de 30\% apresentarem dilatação) em pesquisa com 20 cães em que se fez reparação de ureterotomia de 1,5 a $4 \mathrm{~cm}$ de extensão usando a face seromuscular de alça delgada ou grossa, sem a descontinuidade do trânsito intestinal.

$\mathrm{O}$ achado histológico de epitelização completa nas extremidades do selo ileal, nos grupos A e B, associada à ausência ou lentidão na área central, é altamente sugestivo que o processo de epitelização não é decorrente de metaplasia, mas do crescimento do epitélio ureteral sobre a base de serosa ileal. Esta observação constatada no subgrupo BI (cães sacrificados no $15^{\circ}$ dia de pós-operatório) provavelmente se deve ao maior comprimento do selo. Nada obstante, deve-se assinalar que em todos os animais do subgrupo BII, com evolução de 60 dias, todos os selos de $6 \mathrm{~cm}$ estavam completamente epitelizados. Este achado corrobora a recomendação de Shoemaker (1955) ${ }^{22}$ de manutenção de cateter de moldagem por mais de 14 dias para permitir crescimento epitelial adequado, prevenindo assim estenoses ao nível do enxerto.

A epitelização dos tubos ileais reproduziu o observado com os selos. Observou-se maior facilidade de epitelização nos enxertos mais curtos, pois quando se avaliou histologicamente tubos ileais de $2 \mathrm{~cm}$ (grupo C) a epitelização foi total em $4 / 6$ cães enquanto no grupo $\mathrm{D}(6 \mathrm{~cm})$ a epitelização foi total em $1 / 7$ cães. Nos casos onde a epitelização foi incompleta, principalmente nos do grupo E, em sua totalidade, notou-se por meio de corte longitudinal que a epitelização foi total nos locais mais próximos à anastomose uretero-ileal, sendo que nas porções mais centrais, foram observados apenas focos iniciais do processo de epitelização.

Nos grupos onde se substituiu o ureter por tubo ileal invertido, notou-se que nos cães onde houve obstrução da luz ureteral o tubo de moldagem havia migrado mostrando que, ao contrário do observado nos grupos de selo ileal, onde não foi utilizado tubo de modelagem após o término da sutura, a ausência do tubo favorece a estenose cicatricial. A urográfica 
excretora dos grupos com tubo ileal mostrou que somente 1 animal do grupo CI, 2 do CII, 2 do DI, 1 do DII,1 do EI e 1 do E II apresentavam trato urinário normal. Esses resultados não são favoráveis e não recomendam o método.

Em relação ao muco observado, notou-se que a não ocorrência de coleção de muco no Grupo E indica que a dissecção romba foi mais eficiente que a curetagem para e remoção de mucosa ileal sendo o processo recomendado ao se usar íleo invertido.

A variação do comprimento do tubo ileal invertido foi idealizada no sentido de se avaliar se os resultados seriam afetados por este parâmetro . Nos trabalhos de Shoemaker \& Bower $(1955)^{25}$ foram utilizados longos tubos ileais invertidos anastomosados diretamente no bacinete e na bexiga, sendo que a maioria dos cães apresentou estenose da anastomose proximal atribuída à remoção precoce do cateter ureteral e à irritação provocada pela urina na serosa ileal. Neste estudo apresentado, observou-se estenose cicatricial em 2/4 dos cães com tubos de $2 \mathrm{~cm}$, em $2 / 4$ com tubo de " $6 \mathrm{~cm}$ ( D II) e em 1/4 com tubos de $6 \mathrm{~cm}$ ( E II). Esses dados sugerem que o comprimento do tubo não é fator fundamental para estenose cicatricial e que a manutenção do cateter ureteral por 15 dias seria suficiente para garantir a patência tubular e das anastomoses em pelo menos $50 \%$ dos casos ao final de 2 meses.

\section{CONCLUSÕES}

A substituição ureteral por íleo invertido permite as chegar às conclusões seguintes: 1 - a serosa ileal é recoberta por urotélio na maioria dos cães em período variável de 15 a 60 dias; 2 - o urotélio neo-formado é resultado de crescimento epitelial sobre a serosa do íleo invertido; 3 - Ocorre obstrução mecânica ou funcional em proporção significativa de casos o que descredencia o íleo invertido como substituto ideal do ureter.

\section{REFERÊNCIAS}

1. Harvard BM, Camilleri JA, Nadig PW, Glenn JF. Experimental transplantation of freeze-dried homologous and autologous ureteral segments. J Urol 1961; 86: 385-9.

2. Dufour B, Auvert J. Le replacement de l'uretère par une prothèse d'elastomère de silicone. J Urol Nephrol 1971; 77: 441-50.

3. Rosenberg ML, Dahlen GA. Autogenous vein grafts and venous valves in ureteral surgery: an experimental study. J Urol 1953; 70: 434-6.

4. Hovnanian AP, Kingsley IA. Reconstruction of the ureter by free autologous bladder mucosa graft. J Urol 1966; 96: 167-73.
5. Hardin CA. Experimental repair of ureters by polyethylene tubing and ureteral and vessel grafts. Arch Surg 1954;68:57-61.

6. Warren JWJr, Coomenr T, Fransen H. The use of teflon grafts for replacement of ureters. J Urol 1963; 89: 164-6.

7. Varady S, Friedman E, Yap WT, Lage A, Richie J. Ureteral replacement with a new synthetic material: Gore-Tex. J Urol 1982; 128: $171-5$

8. Itiberê, JLM, Brenner S, Goto M, Netto AGF, Arruda RM. Emprego de enxertos arteriais conservados em álcool na substituição do ureter. Estudo experimental. Rev Paul Med 1959; 54: 25-8.

9. Tachibana M, Nagamatsu RG, Addonizio JC. Ureteral replacement using collagen sponge tube grafts. J Urol 1985; 133: 866-9.

10. Thuroff JW, Hutschenreiter G, Froneberg D, Hohenfellner R. Transplantation of free peritoneal patch in surgery of the renal pelvis and ureter. Eur Urol 1981; 7: 746-57.

11. Klippel KF, Hohenfellner R. Umbilical vein as ureteral replacement. Invest Urol 1979; 16: 447-50.

12. Schein CJ, Sanders AR, Hurwitt ES. Experimental reconstruction of ureters. Substitution with autogenous pedicled Fallopian tube grafts. AMA Arch Surg 1956; 73: 47-53.

13. Masson JC, Mariano A. Remplacemente de l'uretère pelvien gauche par l'appendice. J. Urol. Nephrol 1973; 79: 900-4.

14. Boxer RJ, Johnson SF, Herich RM. Ureteral substitution. Urology 1978; 12: 269-78.

15. Hovnanian AP. Ureteral replacements. Surg Gynecol Obst 1972; 135: 801-10

16. Amin HA. Experience with the ileal ureter. Br J Urol 1976; 48: $19-26$

17. Baum WC. The clinical use of terminal ileum as a substitute ureter. J Urol 1954; 72: 16-33.

18. Goodwin WE, Winter CC, Turner RD. Replacement of the ureter by small intestine: clinical application and results of the ileal ureter. J Urol 1959; 81: 406-18.

19. Fritzsche P, Skinner DG, Crave JD, Goodwin WE. Long-term radiographic chages of the kidney following the ileal ureter operation. J Urol 1975; 114: 843-7.

20. Swenson O, Fisher JH, Cendron J. Megaloureter: investigation as to the cause and report on the newer forms of treatment. Surgery $1956 ; 40$ : 223-33.

21. Turner RD, Goodwin WE. Experiments with intussuscepted ileal valve in ureteral substitution. J Urol 1959; 81: 526-9.

22. Shoemaker WC. Reversed seromuscular grafts in urinary tract reconstruction. J Urol 1955; 74: 453-78.

23. Torbey K, Leadbetter WF. The use of the seromuscular layer of an ileal loop for ureteral replacement. J Urol 1962; 88: 746-57.

24. Kendall RA, Karafin L. Seromuscular ureteroplasty. J Urol 1969; 101: 28-32.

25. Shoemaker WC, Bower R. The surgical reconstruction of the ureter by anew technique. S Forum 1955; 615-19.

\section{Endereço para correspondência:}

Antonio Carlos Pereira Martins

Faculdade de Medicina de Ribeirão Preto - USP, Divisão de Urologia

Av. Bandeirantes n ${ }^{\circ} 3900$, Ribeirão Preto, SP

CEP- 14048-900

e-mail : acpmartins@convex.com.br 